Journal for

ImmunoTherapy of Cancer

\section{Immunotherapy for sarcomas: new frontiers and unveiled opportunities}

To cite: Birdi HK, Jirovec A, Cortés-Kaplan S, et al. Immunotherapy for sarcomas: new frontiers and unveiled opportunities. Journal for ImmunoTherapy of Cancer 2021;9:e001580. doi:10.1136/ jitc-2020-001580

$\mathrm{HKB}, \mathrm{AJ}$ and SC-K contributed equally.

J-SD and MA contributed equally.

Accepted 15 November 2020

Check for updates

(C) Author(s) (or their employer(s)) 2021. Re-use permitted under CC BY-NC. No commercial re-use. See rights and permissions. Published by BMJ.

${ }^{1}$ Cancer Therapeutics Program, Ottawa Hospital Research Institute, Ottawa, Ontario, Canada

${ }^{2}$ Department of Biochemistry, Microbiology and Immunology, University of Ottawa, Ottawa, Ontario, Canada

${ }^{3} \mathrm{Cl} 3$, University of Ottawa,

Ottawa, Ontario, Canada

${ }^{4}$ Department of Surgery, University of Ottawa, Ottawa, Ontario, Canada

${ }^{5}$ Clinical Epidemiology Unit, Ottawa Hospital Reseach Institute, Ottawa, Ontario, Canada

Correspondence to

Dr Michele Ardolino;

mardolino@ohri.ca

\author{
Harsimrat Kaur Birdi, ${ }^{1,2,3}$ Anna Jirovec, ${ }^{1,2,3}$ Serena Cortés-Kaplan, ${ }^{1,2,3}$ Joel Werier, ${ }^{4,5}$ \\ Carolyn Nessim, ${ }^{1,5}$ Jean-Simon Diallo, ${ }^{1,2,3}$ Michele Ardolino (1) 1,2,3
}

\begin{abstract}
Sarcomas are a rare malignancy of mesenchymal tissues, comprizing a plethora of unique subtypes, with more than 60 types. The sheer heterogeneity of disease phenotype makes this a particularly difficult cancer to treat. Radiotherapy, chemotherapy and surgery have been employed for over three decades and, although effective in early disease (stages I-II), in later stages, where metastatic tumors are present, these treatments are less effective. Given the spectacular results obtained by cancer immunotherapy in a variety of solid cancers and leukemias, there is now a great interest in appliying this new realm of therapy for sarcomas. The widespread use of immunotherapy for sarcoma relies on immuno-profiling of subtypes, immunomonitoring for prognosis, preclinical studies and insight into the safety profile of these novel therapies. Herein, we discuss preclinical and clinical data highlighting how immunotherapy is being used in soft tissue sarcoma and bone sarcomas.
\end{abstract}

\section{INTRODUCTION}

Sarcoma is a rare disease of tissues of mesenchymal origin encompassing bone, fat, joint and muscle, ${ }^{1}$ divided in two major types: $>50$ subtypes of soft-tissue sarcoma (STS) and three major subtypes of bone sarcoma (osteosarcoma, chondrosarcoma and Ewing's (EW) sarcoma). ${ }^{23}$ Although rare in adults (annual incidence $<1 \%$ ), sarcomas account for $20 \%$ of all pediatric cancers in North America. ${ }^{4}$ Despite its heterogeneous origins, histology and genetic markers, a common feature of sarcoma is a poor prognosis in patients with advanced disease. Although survival rates for broad sarcoma subtypes are difficult to ascertain due to disease heterogeneity, in general, patients with localized disease benefit from radiotherapy and surgery (5-year survival rate $>80 \%$ in STS patients and $\sim 70 \%$ in bone sarcoma patients). However, patients exhibiting stage III/IV STS or various bone sarcomas have 5-year survival rates of $<20 \%$ and between $22 \%$ and $57 \%$, respectively. ${ }^{4-6}$ Additionally, disease relapse, which occurs in $40 \%-60 \%$ of high grade cases, is not uncommon. ${ }^{7}$ Despite recent advancements in testing, diagnosis, molecular characterization and combination chemotherapies, there has been little progress in improving outcomes in an advanced disease setting.

Cancer immunotherapy has progressed exponentially in recent decades, owing greatly to an improved understanding of the interplay between the immune system and cancer. Immunotherapies such as adoptive cell transfer, oncolytic viruses (OVs) and immune checkpoint blockade (ICB) show promise for the future of sarcoma therapy. However, therapies that have gained traction for the treatment of other cancer types encounter challenges in sarcoma due to: (1) a lack of well established antigens in subtypes that can be targeted by vaccines, therapeutic antibodies or chimeric antigen receptors (CAR) therapy, (2) presence of extensive tumor heterogeneity and (3) a lack of characterization of the tumor microenvironment (TME) in unique subtypes. Herein, we review the various immunotherapeutic strategies pursued for sarcoma for overcoming these challenges.

\section{IMMUNE CHECKPOINT BLOCKADE}

Immune checkpoint receptors are inhibitory molecules expressed on the surface of immune cells, cancer cells and other supporting cells in the TME. This includes molecules like CTLA-4, PD-1, PD-L1, LAG-3, TIM-3 and VISTA. ${ }^{8}$ ICB is used to block receptor-ligand interactions in order to restore anti-tumor immune functions. Monoclonal antibodies targeting CTLA-4, PD-1 and PD-L1 received Food and Drug Administration (FDA)-approval in cancer. ${ }^{9}$

Expression of checkpoint receptors varies widely in sarcoma patients and according to subtype. ${ }^{10-13}$ A study evaluating 1072 sarcoma specimens found that PD- 1 and PD-L1 were expressed in only $10 \%$ and $22 \%$ of cases, meanwhile LAG-3 and TIM-3 were expressed in $42 \%$ and $54 \%$ of cases, respectively. ${ }^{11}$ A higher expression of checkpoint receptors was observed in non-translocationassociated sarcomas, such as dedifferentiated 
liposarcoma (DDLPS), undifferentiated pleomorphic sarcoma (UPS), myxofibrosarcoma and leiomyosarcoma, than translocation-associated sarcomas. ${ }^{11}$ Other groups have also shown that UPS, leiomyosarcoma and LPS patients have increased expression of PD-1/PD-L1, suggesting that these sarcoma subtypes could potentially respond to ICB treatment. ${ }^{10} 14$

Several individual case reports have shown that sarcoma patients treated with ICB achieve a positive response or complete remission. ${ }^{15-19}$ All patients presented with unique sarcoma subtypes and with varying levels of checkpoint receptor expression, thus identifying commonalities between the positive responses was challenging. The SARC028 (NCT02301039) was one of the first multicenter phase II trials evaluating the effectiveness of ICB in sarcoma. ${ }^{20}$ This study comprised of 80 evaluable patients, 40 with STS and 40 with bone sarcoma that were treated with pembrolizumab (anti-PD-1). Response to therapy was limited to patients in the STS cohort with seven patients showing an objective response rate, with greater benefit in UPS and DDLPS patients, whereas little to no benefit in the bone sarcoma cohort was observed. ${ }^{20}$ Tumor biopsies before and during treatment were obtained from patients enrolled in the SARC028 trial to characterize immune features associated with treatment response. ${ }^{21}$ At baseline, higher density of tumor-infiltrating T cells and TAMs expressing PD-L1 were observed in responders versus non-responders. ${ }^{21}$ Sarcoma immune class (SIC) classifications of patients confirmed that tumors in the immune high class (SIC E) showed the most benefit in response to pembrolizumab. ${ }^{22}$ To further investigate the effectiveness of pembrolizumab treatment in patients with STS, the SARC028 study enrolled an expansion cohort to include additional patients; 40 with UPS and 40 with LPS. ${ }^{23}$ The UPS cohort reached its primary endpoint, with an objective response rate in $9 / 40$ patients (two complete and seven partial responses). The LPS group had an objective response rate in $4 / 39$ patients (four partial responses) ${ }^{23}$

The Alliance A091401 study (NCT02500797) evaluated the effectiveness of nivolumab (anti-PD-1) alone or in combination with ipilimumab (anti-CTLA-4) for the treatment of unresectable and metastatic sarcoma, ${ }^{24}$ where 2/38 patients in the nivolumab group and 6/38 patients in the combination group responded. These responses were observed in patients with leiomyosarcoma, UPS, myxofibrosarcoma, angiosarcoma, alveolar soft part sarcoma (ASPS) and malignant fibrous histiocytoma. The clinical benefit of nivolumab monotherapy (median PFS 2 months) was below that of standard chemotherapy and the combination of nivolumab and ipilimumab (median PFS 4 months) was on par with currently available chemotherapy. ${ }^{24}$ Three expansion cohorts for UPS, DDLPS and gastrointestinal stromal tumor were subsequently enrolled for the A091401 study. Only the combination arm for UPS and DDLPS met the primary endpoint (6 months confirmed response rate). Correlative analyzes for genomic and clinical biomarkers and response is currently underway. ${ }^{25}$
Based on the results from the SARC028 trial, another group has begun to recruit patients with UPS and LPS to evaluate the effectiveness of ICB in neoadjuvant settings. ${ }^{26}$ In this phase II, single-center, open-label, randomized non-comparative trial (NCT03307616), patients will receive nivolumab (anti-PD-1) alone or in combination with ipilimumab (anti-CTLA-4) before surgery. The UPS cohort will also receive standard of care radiation therapy for UPS in the trunk/extremities. Interestingly, radiation therapy in UPS increased tumor infiltrating immune cells and tumor PD-L1 expression opening an opportunity to use PD-1/L1 blockade. ${ }^{27}$ Preliminary results from 24 evaluable patients (9 UPS and 14 LPS) show a median pathological response of $95 \%$ in the UPS cohort and $22.5 \%$ in the LPS cohort. ${ }^{28}$ This study is still recruiting and will also include transcriptome, immune and microbiome profiling, which will provide much needed information about the immune response in sarcoma and the molecular mechanisms that promote/hinder the response to ICB in UPS and LPS. ${ }^{26}$ So far, these highlighted studies show that UPS and LPS are responsive to ICB. These tumors have an increased amount of tumor infiltrating immune cells and expression of PD-1/PD-L1 which could be a contributing factor their responsiveness. ${ }^{26}$

ICB has been also combined with chemotherapy or targeted therapy. A phase I/II trial (NCT02888665) assessed the efficacy of pembrolizumab plus doxorubicin in patients with metastatic and/or unresectable sarcoma. Although, this study did not meet its primary response rate endpoint, the combination therapy was well tolerated and doubled the median progression-free survival. ${ }^{14}$ A phase II study (NCT02636725) evaluated the efficacy of combining pembrolizumab and axitinib, a VEGFR inhibitor, in patients with advanced metastatic sarcoma. $65.6 \%$ of treated patients met the primary endpoint (3 months progression-free survival) with most responses occuring in patients with ASPS. ${ }^{29}$ Notably, tumor biopsies from ASPS patients showed high tumor infiltrating lymphocyte (TIL) infiltration and PD-L1 expression, suggesting a 'hot' immune phenotype. This could potentially explain why ASPS responded to ICB, when typically tumors with low mutational burden such as ASPS are associated with poor ICB responsiveness. ${ }^{29}$ These studies highlight the need for improved treatment for patients with advanced metastatic and unresectable sarcomas.

\section{Adoptive cell therapy}

T cells

Adoptive cell therapy (ACT) involves the extraction of immune cells from a patient, or from healthy donors, which are then manipulated ex vivo and expanded prior to reinfusion in the patient. Promising results in hematological and some solid malignancies have been achieved by using $\mathrm{T}$ cells transduced with vectors encoding $\mathrm{T}$ cell receptors (TCRs) recognizing HLA I-restricted antigens or CARs recognizing cell surface proteins. ${ }^{30}{ }^{31}$ In both cases, the antigen recognized by the exogenous TCR or CAR is expressed uniquely, or preferentially, by tumor 
cells (figure 1). This represents a major obstacle in the expansion of these therapeutic strategies for sarcoma, as these tumors are highly heterogeneous and, as such, also vary greatly in their antigenic landscape both between subtypes and within a single disease.

Expression of cancer testis antigens (CTAs), including MAGE, NY-ESO-1 and SSX, is restricted to the germline, but these molecules are also broadly upregulated in various tumors. ${ }^{32}$ Expression of either/both NY-ESO-1 or/and MAGE-A4 has been observed in $>50 \%$ of primary synovial sarcoma specimens, with NY-ESO-1 expression correlating with better 5 -year overall survival rates. ${ }^{33}$ Varying degrees of NY-ESO-1 expression, and co-expression with MAGE-A4, has also been observed in myxoid LPS, osteosarcomas, pleomorphic LPS and chondrosarcomas ${ }^{34}$ highlighting CTAs as appealing targets for ACT in sarcoma. In a pilot trial, treatment with transgenic $\mathrm{T}$ cells targeting NY-ESO-1, in combination with interleukin (IL)-2, resulted in objective clinical responses in $60 \%$ of refractory synovial sarcoma patients, ${ }^{35}$ which was later corroborated in metastatic synovial sarcoma where treatment led to tumor regression in 50\% of patients. ${ }^{36}$ Promisingly, patients expressing both low or high levels of NY-ESO-1 responded to therapy. ${ }^{37}$

\section{CAR T Cells}

Differently than TCRs, that can only recognize MHC class I restricted peptides, CARs can target any protein expressed on the surface of tumor cells, and are therefore a primary focus for ACT. The identification of tumorspecific antigens for CAR targeting has historically been a hurdle to the implementation of these therapies in solid tumors, but recent preclinical studies have identified promising targets in sarcoma, including GD2 expressed in human osteosarcoma, rhabdomyosarcoma (RMS) and EW sarcoma, B7-H3 (CD276) expressed in pediatric solid sarcomas, and Platelet-derived growth factor receptor $\alpha$ expressed in RMS and HER2-expressed in osteosarcoma (figure 1). ${ }^{38-43}$

However, the promising in vitro results showing that GD2-specific CAR T cells lysed GD2+ human sarcoma cell lines and patient-derived primary osteosarcoma tumor cells, have not been met with successful translation in preclinical models, where GD2-specific CAR $\mathrm{T}$ cells failed to control growth of xenograft tumor models. ${ }^{44}$ However, the administration of drugs that target MDSCs or the protumorigenic factor HFG increased the therapeutic efficacy of GD2-specific CAR T cells, suggesting that in sarcoma CAR $T$ cells need additional help to overcome the barriers in the TME. ${ }^{40}{ }^{45}$ To partially address this point, alternative effectors to $\mathrm{T}$ cells have been tested as recipients of the CAR vector, including cytokine induced killers cells, a heterogeneous population comprised of natural killer T cells (NKT), cytotoxic T cells and NK cells, which showed promising results in vitro and in vivo. ${ }^{46} 47$

The first CAR $\mathrm{T}$ cell therapy evaluated in sarcoma patients (16 osteosarcomas, $1 \mathrm{EW}$ sarcoma) was a first generation HER2-targeting CAR. This phase I study showed safety and partial efficacy with $4 / 17$ patients with stable disease for up to 14 months. CAR-T cells were detected in tumors of 2/2 patients examined, even if no radiologic complete responses were observed. ${ }^{48}$ To improve on these encouraging results, clinical studies are now evaluating targeting different antigens (eg, receptor tyrosine kinase-like orphan receptor 2, CD133, GD-2, Muc1 and CD117) and the use of second-generation, thirdgeneration and fourth-generation CARs (NCT03618381, NCT03356782, NCT01953900) ${ }^{49}$ Ongoing ACT clinical trials using endogenous, transgenic or CAR $\mathrm{T}$ cells are listed in table 1.

\section{NK cells}

Alternatively to T cells, NK cells can be used in ACT, as established successfully in xenograft sarcoma mouse models and in a first-in-dog clinical trial. Ten dogs with advanced osteosarcoma received radiation in combination with intravenous NK cell transfer. Treatment was overall well tolerated and five patients remained metastasis-free at the 6-month primary endpoint. ${ }^{50-53}$ Most clinical trials involving adoptive NK cell therapy are investigating their use for hematological malignancies, however, studies are emerging for their use in solid tumors. Clinical trials for NK cell therapy with published results are reviewed here. ${ }^{54}$ There are several active and recruiting clinical trials investigating the use of NK cell therapy in sarcoma (table 1). A phase I trial (NCT02890758) is evaluating the use of universal donor NK cells for patients with various cancer indications including STS, RMS and EW. Usually for NK cell therapy, HLA-matched donor NK cells are used. In this trial, unmatched healthy donor NK cells are used in combination with ALT803, a protein that supports NK cell growth and viability (figure 1). Interestingly, aerosol delivery of IL-2, in combination with adoptive NK cell transfer in preclinical models, showed enhanced therapeutic effects of NK cells in delaying or completely eliminating lung metastasis in a model of osteosarcoma. Considering the approval of IL-2 for other cancers, aerosol delivery of IL-2 in osteosarcoma patients should be considered for ACT. ${ }^{55}$

\section{Vaccines}

Therapeutic cancer vaccines are designed to stimulate a patient's immune system against cancer and typically target a tumor antigen. Many approaches can be taken to elicit this anti-tumor immune response, such as a peptide vaccine incorporating tumor antigen peptide(s) combined with an adjuvant. A phase II trial of a trivalent ganglioside vaccine (GM2, GD2 and GD3) in 136 sarcoma patients observed serological GM2-specific and GD2-specific responses in 98\% of treated patients, however, no difference in progression-free survival was observed between patient cohorts (NCT01141491) (figure 1) ${ }^{38}$ Similarly, in a study using a 9-mer peptide spanning the SYT-SSX junction in 21 synovial sarcoma patients, 7 patients showed a twofold increase in SYT-SSX 


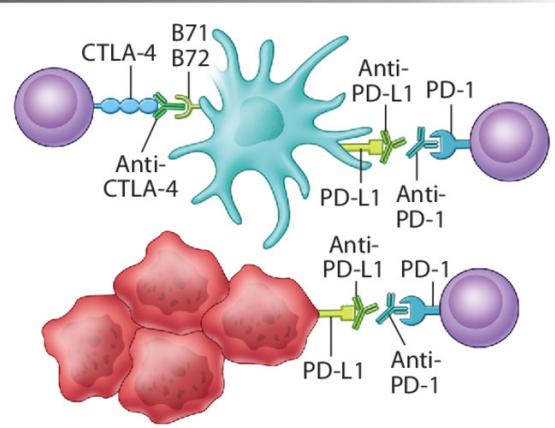

3

\section{CAR-T Cells}

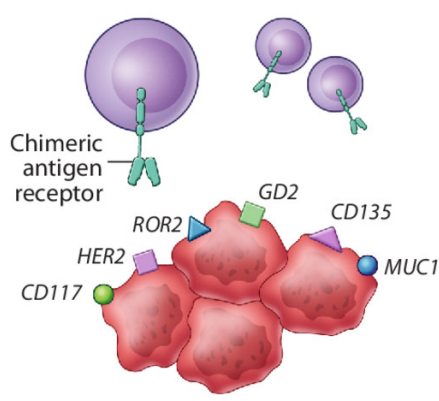

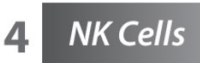

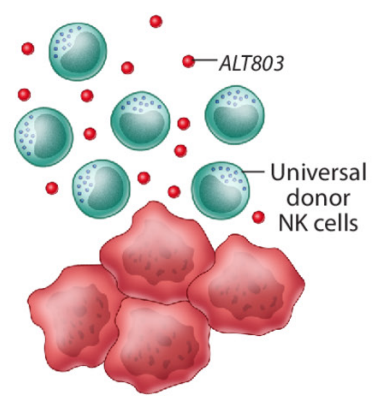

2

Adoptive T Cells

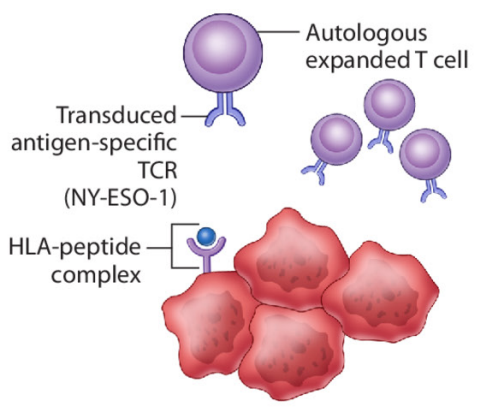

5 Vaccines

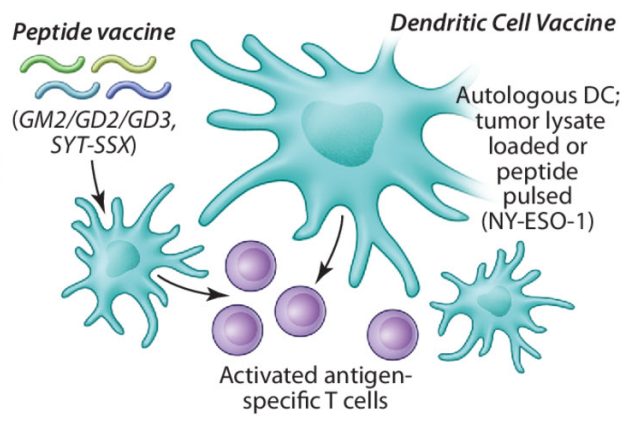

7 Antibodies

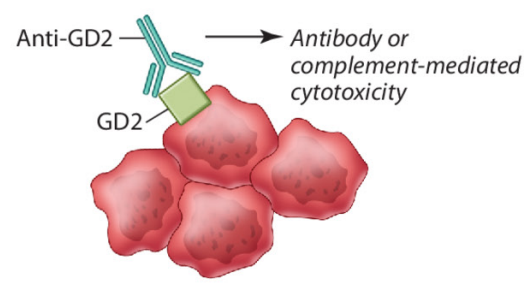

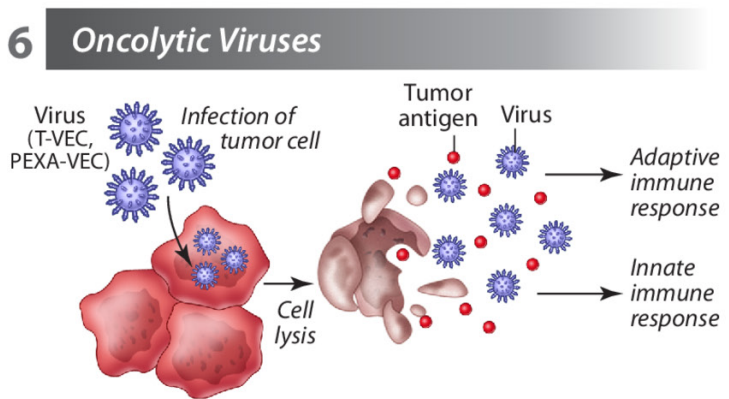

Figure 1 Harnessing the immune system against sarcoma. (1) Immune checkpoint inhibitors restore the ability of immune cells (eg, cytotoxic T cells) to mediate anti-cancer immunity. (2) Autologous T cells transduced with a T cell receptor specific for tumor antigens are expanded and reinfused in patients where they exert cytotoxic functions. (3) CAR T cells against several sarcoma specific antigens can be designed to further boost adoptive T cell therapy. (4) Universal donor NK cells administered with ALT803 may enhance the therapeutic effects of NK cells. (5) Therapeutic cancer vaccines are designed to stimulate a patient's immune system against cancer and typically target a tumor antigen. Vaccine approaches evaluated in sarcoma include peptide vaccines, or dendritic cell vaccines loaded with tumor lysate or pulsed with antigenic peptide. (6) Oncolytic viruses T-VEC and Pexa-VEC are being evaluated in sarcoma, and promote tumor destruction by direct lysis of tumor cells, transgene expression (eg, GM-CSF) and stimulation of adaptive and innate immune responses. (7) Anti-tumor immunity can be promoted by antibodies targeting tumor antigens (eg, GD2) eliciting antibody-mediated or complement-mediated cytotoxicity. (8) Molecules preventing polarization of anti-cancer M1 macrophages to tumor-promoting M2 macrophages can potentiate anti-cancer immunity in sarcoma. CAR, chimeric antigen receptors; CSF1R, colony stimulating factor 1 receptor; GM-CSF, granulocyte-macrophage colony-stimulating factor; NK, natural killer; T-VEC, talimogene laherparepvec. 


\begin{tabular}{|c|c|c|c|c|}
\hline Intervention & Disease & Status & Clinical trial ID & Locations \\
\hline $\begin{array}{l}\text { TIL } \\
\text { IL-2 } \\
\text { Fludarabine } \\
\text { Cyclophosphamide }\end{array}$ & High grade STS & Phase I, Recruiting & NCT04052334 & USA \\
\hline NY-ESO- $1^{\mathrm{c} 259} \mathrm{~T}$ cells & Myxoid/round cell liposarcoma & Phase II, Recruiting & NCT02992743 & USA \\
\hline $\begin{array}{l}\text { Engineered NY-ESO-1 (GSK3377794) } \\
\text { T cells } \\
\text { Fludarabine } \\
\text { Cyclophosphamide }\end{array}$ & Synovial Sarcoma & Phase II, Recruiting & NCT03967223 & USA \\
\hline $\begin{array}{l}\text { ADP-A2M4 SPEAR anti-MAGE-A4 } \\
T \text { cells }\end{array}$ & $\begin{array}{l}\text { Synovial Sarcoma, } \\
\text { Myxoid/round cell liposarcoma }\end{array}$ & Phase II, Recruiting & NCT04044768 & USA \\
\hline $\begin{array}{l}\text { Anti-GD2 (C7R-GD2.CAR) T cells } \\
\text { Fludarabine } \\
\text { Cyclophosphamide }\end{array}$ & $\begin{array}{l}\text { Relapsed OS, relapsed Ewing's } \\
\text { sarcoma, relapsed RMS }\end{array}$ & Phase I, Recruiting & NCT03635632 & USA \\
\hline GVAX & $\begin{array}{l}\text { Clear Cell Sarcoma, } \\
\text { Alveolar Soft Part sarcoma }\end{array}$ & Phase I, Active & NCT00258687 & USA \\
\hline MVA expressing p53 & STS & Phase I, Active & NCT02432963 & USA \\
\hline NK cell infusion HSCT & RMS & Phase I, II, Completed & NCT01386619 & $\begin{array}{l}\text { Germany } \\
\text { Switzerland }\end{array}$ \\
\hline $\begin{array}{l}\text { NK cell infusion and recombinant } \\
\text { IL-15 }\end{array}$ & Sarcoma & $\begin{array}{l}\text { Phase I, } \\
\text { Completed }\end{array}$ & NCT01875601 & USA \\
\hline Expanded, activated NK cells & Ewing Sarcoma, RMS & Phase I, II, Recruiting & NCT02409576 & Singapore \\
\hline NK cell infusion HSCT & $\begin{array}{l}\text { Ewing Sarcoma, OS, RMS, } \\
\text { STS }\end{array}$ & $\begin{array}{l}\text { Phase II, Active, not } \\
\text { recruiting }\end{array}$ & NCT01807468 & South Korea \\
\hline
\end{tabular}

CAR, chimeric antigen receptor; DC, dendritic cell; GD2, disialoganglioside; HSCT, hematopoietic stem cell transplantion; IL-15, interleukin 15; MVA, modified vaccinia Ankara; OS, osteosarcoma; RMS, rhabdomyosarcoma; STS, soft-tissue sarcoma; TIL, tumor infiltrating lymphocyte.

specific CD8+T cells, but only one patient showed transient shrinkage of a metastatic lesion. ${ }^{56}$

The lack of therapeutic response using the aforementioned peptide vaccine approaches in sarcoma prompted the development of alternative vaccination strategies. Dendritic cell (DC) vaccines consist of patient-derived DCs loaded with autologous tumor lysate or tumor antigens ex vivo. An ongoing phase I study is investigating a tumor-lysate loaded monocyte-derived DC vaccine in advanced sarcoma and neuroblastoma pediatric patients (EudraCT 2014-003388-39). Forty-seven pediatric patients have been enrolled in the study, 11 with sarcoma. The treatment was safe and immunomonitoring of nine sarcoma patients (two EW sarcoma, four osteosarcoma, one synovial sarcoma and two RMS) revealed enhanced ex vivo $\mathrm{T}$ cell responses on vaccination. ${ }^{5758}$ In another phase I/II trial, a tumor lysate-pulsed DC vaccine was evaluated in 37 patients with bone or STS. No adverse effects were associated with treatment, and immunological responses to the treatment included significantly elevated interferon (IFN- $\gamma$ ) and IL-12 in the serum, suggesting that this vaccine activated the patient's immune response. However, despite evidence of boosted immunity, only $1 / 35$ patients achieved a partial response to therapy. ${ }^{59}$
Another approach to DC-vaccination is to peptide-pulse DCs with a tumor antigen, forgoing the need for invasive surgical resection of tumor to isolate tumor lysates. One pilot study, including patients with advanced sarcoma that have progressed on standard-of-care chemotherapy and radiation (including synovial sarcoma, osteosarcoma and LPS), uses NY-ESO-1 peptide-pulsed DCs in combination with IL-2 and autologous lymphocytes tranduced with a NY-ESO-1-specific T-cell receptor, with or without CTLA-4 blockade (NCT02070406, NCT01697527). ${ }^{60}$ Treatments were safe but, only one synovial sarcoma patient (receiving treatment without iplimumab) progressed to complete response, despite evidence of anti-tumor activity determined by positron emission tomography imaging in 6 out of 10 patients. This lack of long-lasting response to therapy in the majority of patients could be attributed to the fact that the adoptively transferred cells were found to be short lived in peripheral circulation.

One preclinical study employs a conjugate vaccine targetting the tumor vasculature, thus overcoming the issues associated with targetting tumor antigen. Prophylactic immunization against the tumor vascular marker ED-B (extra domain-B) of fibronectin reduced murine fibrosarcoma (T241) tumor micro vessel density, increased the number of tumor infiltrating leukocytes, 
and generated a high anti-EDB antibody titer compared with controls. ${ }^{61}$ This promising technology can be used for the induction of immune responses against other tumor-antigens or components of the TME and may be an improvement on traditional peptide vaccines.

Although cancer vaccines for sarcoma appear to be safe and result in an immunological response in most of the patients, limited improvement in clinical outcome of patients suggests that many modifications need to be made to attain better therapeutic outcomes. Additional clinical trials using vaccines for sarcoma are listed in table 1 .

\section{Oncolytic viruses}

OVs are self-propagating viruses that selectively infect and kill tumor cells. In addition to their direct effects, OVs promote virally encoded transgene expression (eg, cytokines), cause vascular collapse, and generally alter the TME to stimulate antitumor immune responses. Pointing toward the possibility of using OVs in sarcoma immunotherapy, a comprehensive study comparing the efficacy of four OV head to head in a panel of sarcoma cell lines showed that the rhabdoviruses Maraba and vesicular stomatitis virus (VSV) were highly effective at broadly killing sarcoma cell lines, while Reovirus and herpes simplex virus type I (HSV) were only effective in EW sarcoma cell lines, and synovial sarcoma (SW982) was resistant to infection by all viruses tested.$^{62}$ This result corroborates previous findings that SW982 cells are refractory to infection by a different VSV strain. ${ }^{63}$ Results obtained with cell lines were corroborated using ex vivo infection of freshly collected human sarcoma samples where Maraba successfully infected $\sim 95 \%$ of samples. Encouragingly, mice injected subcutaneously with the sarcoma cell line S180 responded to Maraba treatment, revealing Maraba MG1 as a promising candidate for sarcoma immunotherapy. ${ }^{63}$

When tested in vitro, $\mathrm{H}-1 \mathrm{PV}$, a rodent protoparvovirus non-pathogenic in humans, effectively infected human synovial sarcoma ( $\mathrm{Sw}-\mathrm{R}$ ), a subtype that is resistant to VSV infection, as well as osteosarcoma and EW sarcoma cell lines. ${ }^{63-66}$ However, when evaluated as a therapeutic in a xenograft model, one dose of H-1PV did not lead to tumor regression or increased overall survival, although the lack of therapeutic effect could be attributed to insufficient dosing or owing to the use of immunodeficient mice in this study as OVs work, at least in part, by inducing antitumor immunity. ${ }^{66}$

There is a rich pipeline of OVs in pre-clinical evaluation and many have progressed to clinical trials. Few have gained regulatory approval, such as the genetically engineered HSV-I Talimogene laherparepvec (T-VEC; Imlygic), which has specific virus attenuating mutations and encodes granulocyte-macrophage colony-stimulating factor (GM-CSF) in order to promote anti-tumor immune responses. ${ }^{67}$ Imlygic, was approved for treatment of advanced melanoma by the FDA in 2015. T-VEC in melanoma has led to its evaluation in other solid malignancies, including sarcoma (figure 1).

Two phase II clinical trials evaluating T-VEC are currently recruiting. The first is determining the combination of T-VEC and radiation therapy as a preoperative treatment for patients with newly diagnosed local STS (NCT02923778); the second is evaluating the efficacy of intratumoral administered T-VEC in locally advanced cutaneous angiosarcoma. Another oncolytic HSV-1 (HSV1716) was tested in phase I clinical trial in patients with solid tumors that included eight pediatric patients with RMS, EW sarcoma, osteosarcoma, MPNST or chordoma and two adult patients with chondrosarcoma or EW sarcoma (NCT00931931). Unfortunately, while the virus was found safe, no objective responses were observed, suggesting that HSV-1 may not be ideally suited for these sarcoma subtypes. ${ }^{68}$

Pexastimogene devacirepvec (Pexa-Vec) is derived from a Wyeth vaccinia virus strain and engineered to target cancer cells. Similar to Imlygic, it is armed with GM-CSF. In a phase I trial, Pexa-Vec was safe when administered intratumorally in a cohort of RMS, Ewings sarcoma, osteosarcoma, and other STS pediatric patients (NCT01169584). A phase I/II study of Pexa-Vec in combination with metronomic cyclophosphamide in patients with advanced STS and advanced breast cancer is currently recruiting (NCT02630368).

Overall, preclinical studies suggest OV could be promising immunotherapies for the treatment of sarcoma. However, this remains to be corroborated in clinical studies. So far, OVs have had limited success as monotherapies and it is likely that OVs will require use in combination with other modalities that can overcome known resistance mechanisms, including innate antiviral responses and immunological resistance, for example. ${ }^{69-72}$ Additionaly, some OVs may be more effective than others depending on the sarcoma subtypes as indicated from comparative in vitro studies. ${ }^{62}$

\section{Cancer-targeted antibodies}

Anti-tumor immunity can be promoted by antibodies targeting tumor antigens eliciting antibody-mediated or complement-mediated cytotoxicity. Surface disialoganglioside (GD2), a glycosphingolipid involved in cell proliferation, is also a known tumor associated antigen, most notably expressed in neuroblastoma, melanoma and half of all osteosarcomas and STS'. ${ }^{73}$ Widespread distribution of GD2 in neuroblastoma prompted preclinical development of GD2-targeting mAbs and led to phase I trials of humanized 3F8 (hu3F8) and FDA approval of the mAb ch14.18, also known as dinutuximab, in 2015 (figure 1). ${ }^{745}$ GD2 is expressed in $~ 50 \%$ of osteosarcomas and STS', although with significant intratumoral heterogeneity, therefore, there is a potential of expanding the use of anti-GD2 for osteosarcoma, with clinical trials currently ongoing ${ }^{39} 7376$ (NCT02502786, NCT02484443). 


\section{Targeting macrophages}

The polarization of macrophages associated to the tumor (TAMs), either directly infiltrating the TME or deriving from monocytes, is a key determinant of the outcome of anti-tumor immunity. In a broad sense, M1 macrophages typically promote anti-cancer immunity whereas M2 macrophages are usually associated with worse prognosis given they promote immunosuppression, tumor angiogenesis and metastasis. Therapies that indirectly prevent macrophage polarization toward an M2 phenotypes are currently being tested in preclinical models. For example, treatment of MCA-205 fibrosarcoma mouse model with anti-angiogenic peptide led to a reduction of M2-polarized macrophages, which was important for the therapeutic effect of the peptide. In another study, TAMs were directly targeted by treating sarcoma-bearing mice with nanoparticles containing zoledronate leading to significant tumor regression. ${ }^{77} 78$ A more favorable TAM polarization was instead achieved using TLR2 agonists that increased the M1/M2 ratio in sarcoma-bearing mice leading to decreased tumor growth. ${ }^{79}$

The CD47-SIRP $\alpha$ pathway promotes tumor immune escape by inhibiting macrophage phagocytosis (do not eat me signal) and is an appealing target for immunotherapy as revealed by studies showing that: (1) an abundance of SIRP $\alpha$ in TAMs was associated with worse prognosis in patients with synovial sarcoma and myxofibrosarcoma; and (2) high expression of CD47 correlated with poor survival in osteosarcoma patients. ${ }^{80}{ }^{81}$ In an osteosarcoma mouse model, mice treated with a CD47 blocking antibody, alone or in combination with doxorubicin, had reduced tumor burden. ${ }^{82}$ Although no clinical trials are currently ongoing in sarcoma for monoclonal antibodies targeting CD47, targeting of the same pathway is under clinical evaluation in a phase I trial (NCT02890368) in solid tumors, including STS, using TTI-621, a SIRP1 $\alpha$-fusion protein that blocks CD47 binding to SIRP1 $\alpha$ and simulataneously potentiated macrophage activation by binding to activating Fc receptors. ${ }^{83}$

The CSF1 receptor (CSF1R)/CSF1 axis was considered a promising targetable pathway to reduce M2 TAMs in sarcomas but a phase I/IIa clinical trial assessing the effect of small molecules targeting CSF1R signaling in solid tissue malignancies (NCT02452424) was terminated due to lack of clinical efficacy (figure 1). Evaluation of monoclonal antibodies targeting CSF1R (Emactuzumab) is still ongoing for the treatment of STS with no results posted to date (NCT02323191). In conclusion, targeting the macrophage compartment is a compelling yet still fairly unexplored avenue to potentiate anti-cancer immunity in sarcoma.

\section{Cytokine modulation}

Cytokines play a key role in communication between immune cells and can be exploited to harness their effector functions. To date, IL-2 and IFN- $\alpha$ are the only immunostimulatory cytokines approved by the FDA for the treatment of several cancer indications including
HIV-induced Kaposi's sarcoma, a malignancy of blood and lymph vessels caused by the human herpersvirus $8{ }^{84}{ }^{85}$ As a single agent, IFN- $\alpha$ cytokine therapy is most successful at high doses and in Kaposi's sarcoma patients with higher baseline CD4+ Tcell counts but is commonly used in conjunction with chemotherapies. With the advent of anti-retroviral therapy, IFN- $\alpha$ began to be used in lower doses in combination with these anti-retrovirals. Overall, the anti-proliferative, anti-angiogenic and antiviral effects of IFN- $\alpha$ therapy are thought to induce tumor regression in HIV-induced Kaposi's sarcoma. ${ }^{86}$ However, liposomal doxorubicin and paclitaxel are still the front line treatment for Kaposi's sarcoma.

The transforming growth factor-beta (TGF- $\beta$ ) signaling pathway is known to be dysregulated in many cancers and the cytokine is notorious for its dual role of both tumor promoting and tumor suppressing activities in late stage and early-stage cancers, respectively. ${ }^{8788}$ In the case of osteosarcoma, TGF- $\beta$ was found to be increased in the serum of patients compared with healthy controls and is now seen as a driver of metastatic potential in this cancer. ${ }^{89} 90$ Furthermore, in vitro studies revealed that treatment of osteosarcoma cell lines with TGF- $\beta$ induced epithelialmesenchymal transition, providing further evidence of its role in stimulating metastasis. ${ }^{91} 92$ Decreasing levels of serum TGF- $\beta$ is also seen in EW sarcoma and RMS patients during remission, and as such may have prognostic value.$^{93}$ In light of these findings, if the key role of TGF- $\beta$ is elucidated, there is potential in inhibiting the key components of the TGF- $\beta$ pathway as a therapeutic strategy for osteosarcoma, EW sarcoma and RMS. Clinical trials of TGF- $\beta$ ligand and receptor inhibitors are ongoing for many advanced stage carcinomas, melanomas and gliomas and should, therefore, be given considerable thought for its use in osteosarcoma. ${ }^{8794}$

\section{Combination therapies}

Since ICB has shown limited efficacy as a monotherapy for sarcoma, numerous studies are investigating ICB therapy in combination with other treatments. More specifically, one study evaluated NY-ESO-1 specific CD8 $\mathrm{T}$ cells of patients with NY-ESO-1+ positive tumors and found that these cells are enriched for expression of LAG-3 and PD-1. ${ }^{95}$ To address upregulation of inhibitory molecules, an ongoing study is evaluating a vaccine targeting NY-ESO-1 (called CMB305) in combination with anti-PDL1 (atezolizumab) in sarcoma (NCT02609984). In the context of OV, Newcastle disease virus (NDV) for treatment of syngeneic tumor models of melanoma induced PD-L1 upregulation of infected cells, and inhibited effector functions of tumor infiltrated T cells. ${ }^{71}$ Similarly, oncolytic vaccinia virus treatment of murine models of colon and ovarian cancer induced PD-L1 expression in cancer and immune cells. ${ }^{96}$ Suggesting immune checkpoints are a potential contributor to resistance to $\mathrm{OV}$ therapy, the combination of NDV and other OVs with ICB has shown to provide therapeutic benefit in various preclinical models. ${ }^{71}$ A phase 2 trial (NCT03069378) 
recently investigated the combination of pembrolizumab with T-VEC. ${ }^{97}$ The overall response rate was $35 \%(7 / 20)$ with no complete responses and 7 partial responses. The partial responses were observed in patients with UPS, myxofibrosarcoma, epithelioid sarcoma, cutaneous angiosarcoma and an unclassified sarcoma. Also, the TIL score and PD-L1 expression was higher in responders compared with the refractory group. ${ }^{97}$ An expansion cohort for this study is underway for patients with UPS, myxofibrosarcoma and angiosarcoma. A multi-center pilot study (NCT03282344) is currently evaluating the safety and efficacy of treating refractory sarcoma patients with nivolumab and NKTR-214, an IL-2 pathway agonist. Preliminary results from 50 enrolled patients showed partial responses in patients with UPS $(2 / 10)$, dedifferentiated chondrosarcoma $(1 / 10)$ and leiomyosarcoma $(1 / 10)$ and no respondes from DDLPS and osteosarcoma patients. ${ }^{98}$ A phase II multi-arm study (NCT02815995) investigated the benefit of combining durvalumab (anti-PD-L1) and tremelimumab (anti-CTLA-4). Their primary endpoint which was progression-free survival at 3 months, was reached in $51 \%$ of patients $(n=57) .{ }^{99}$ It was also observed that a high immune infiltration score could predict clinical benefit. Many of the combination ICB trials include patients with advanced sarcomas who are refractory to standard treatment and/or did not respond to previous ICB monotherapy. Due to the nature of these studies, monotherapy groups are not included, which can make it difficult to determine which component of the combination treatment improved the clinical outcome.

\section{Predicting response to ICB immunotherapy}

The clinical studies discussed show that there is a heterogeneous response of sarcoma to checkpoint blockade. Despite the progress of the most promising immunotherapeutic approach, ICB, only a small proportion of patients given this treatment have clinical responses. One consideration is that patient selection for ICB treatment is generally performed in a single analyte manner, whereby patients are selected based on expression levels of immune inhibitory molecules being targeted. As we increase our understanding of the immune mechanisms at play within the tumor, it is evident that this analysis provides an incomplete picture of the dynamic tumor immune microenvironment that contributes to the efficacy of ICB therapy. To this end, several studies have sought to establish predictive biomarkers using multigene analysis to guide treatment.

A seminal study identified an immune-related signature correlating with clinical responses from different clinical studies using pembrolizumab (KEYNOTE-180, KEYNOTE-181, KEYNOTE-158), and developed a scoring algorithm predicting patients' response to pembrolizumab based on tumor expression of 18 IFN- $\gamma$-related immune genes. The Tumor Inflammation Signature (TIS) score measures activated but suppressed adaptive immune responses within tumors. Notably, this score has been retrospectively applied to clinical trial data, and high scores were found to be associated with response to pembrolizumab, ${ }^{100}{ }^{101}$ further supporting the use TIS to guide patient selection in future ICB clinical trials for sarcoma.

Another group developed a new immune-based classification of STS based on the composition of the tumor immune microenvironment determined from gene expression profiles, and stratified 608 STS into one of five SICs with highly distinct immune profiles. Patients with tumors classified in the immune high group-characterized by high expression of genes specific to CD8+ T cells, NK cells, B cells and tertiary lymphoid structures, exhibited the largest response rate to PD-1 blockade therapy (in comparison to tumors with different immune class) in a phase I clinical trial of pembrolizumab in STS (SARC028). This study highlights the heterogeneity of sarcoma, and the need for increased understanding of immune status of sarcomas to select appropriate immunotherapy treatments for patients. ${ }^{22}$

Altogether, these data show that similarly to other cancers, in sarcoma a T cell-inflamed TME, characterized by cytotoxic effector lymphoid functions and active IFN- $\gamma$ signaling, are features of patients responding to PD-1 blockade. Further elucidating the tumor immune microenvironment of sarcoma subtypes will refine patient eligibility criteria for sarcoma immunotherapy trials, and further investigation will be necessary to predict patient outcome in response to other immunotherapeutic approaches discussed in this review.

\section{CONCLUSION}

Recent research has highlighted an opportunity to harness the immune response to develop immunotherapies against sarcoma. There is currently a wealth of immunotherapy strategies that can be employed which include: ICB, ACT, conventional vaccines, OV and targeting of innate immune mechanisms such as macrophages and cytokine modulation therapies. It is often difficult to navigate which therapeutic approach is likely to be more successful. Here, we reviewed preclinical and clinical studies that have focused on investigating which immunotherapy can succeed in treating the different sarcoma indications. While it is difficult to adequately capture the complexity of sarcomas, it appears combination therapies involving ICBs is likely the path forward. As described above, ICB combinations with OVs, antigen targeting vaccines and ACT will provide a holistic path towards treatment. Disease progression and disease recurrence, both prevalent complications of many sarcomas, should also be taken into consideration when choosing a therapy. However, each subtype will nevertheless require stringent characterization of its immune components, biomarkers and response predictors to select an optimal therapy.

Correction notice This article has been corrected since it was first published. The overall response rate reported for reference 97 (Kelly et al) was cited incorrectly and has now been amended. 
Twitter Michele Ardolino @qfkc

Contributors HKB, AJ, SC-K wrote the manuscript. JW and CN edited the manuscript. J-SD and MA wrote the manuscript and supervised the preparation of the review.

Funding Sarcoma research in the Ardolino Lab is funded by CIHR (to MA) and a University of Ottawa Translational Research Grant (to MA and CN). Sarcoma research in the Diallo lab is funded by the Valerie's Flutter foundation, BioCanRx, the Ontario Molecular Pathology Research Network (OMPRN) and a CIHR New Investigator award (Infection and Immunity).

Competing interests J-SD is an inventor on patents licensed to Turnstone Biologics, which is commercializing oncolytic Maraba virus. J-SD has patents licensed and also holds equity in Virica Biotech, which is developing oncolytic virus platforms.

Patient consent for publication Not required.

Provenance and peer review Not commissioned; externally peer reviewed.

Open access This is an open access article distributed in accordance with the Creative Commons Attribution Non Commercial (CC BY-NC 4.0) license, which permits others to distribute, remix, adapt, build upon this work non-commercially, and license their derivative works on different terms, provided the original work is properly cited, appropriate credit is given, any changes made indicated, and the use is non-commercial. See http://creativecommons.org/licenses/by-nc/4.0/.

\section{ORCID iD}

Michele Ardolino http://orcid.org/0000-0003-4114-0985

\section{REFERENCES}

1 Burningham Z, Hashibe M, Spector L, et al. The epidemiology of sarcoma. Clin Sarcoma Res 2012;2:14.

2 Katz D, Palmerini E, Pollack SM. More than 50 subtypes of soft tissue sarcoma: paving the path for Histology-Driven treatments. Am Soc Clin Oncol Educ Book 2018;38:925-38.

3 Ferguson JL, Turner SP. Bone cancer: diagnosis and treatment principles. Am Fam Physician 2018;98:205-13.

4 Cancer Statistics Review, 1975-2017 - SEER Statistics. Available: https://seer.cancer.gov/csr/1975_2017/ [Accessed 5 Jul 2020].

5 Sarcoma, soft tissue: statistics. Available: https://www.cancer.net/ cancer-types/sarcoma-soft-tissue/statistics [Accessed 20 Sep 2020].

6 Bone cancer: statistics. Available: https://www.cancer.net/cancertypes/bone-cancer/statistics [Accessed 20 Sep 2020].

7 Rutkowski P, Ługowska I. Follow-Up in soft tissue sarcomas. Memo 2014;7:92-6 https://pubmed.ncbi.nlm.nih.gov/25089160/

8 Qin S, Xu L, Yi M, et al. Novel immune checkpoint targets: moving beyond PD-1 and CTLA-4. Mol Cancer 2019;18.

9 Lu R-M, Hwang Y-C, Liu I-J, et al. Development of therapeutic antibodies for the treatment of diseases. J Biomed Sci 2020;27:1-30.

10 Yan L, Wang Z, Cui C, et al. Comprehensive immune characterization and T-cell receptor repertoire heterogeneity of retroperitoneal liposarcoma. Cancer Sci 2019;110:3038-48 https:// pubmed-ncbi-nlm-nih-gov.proxy.bib.uottawa.ca/31385405/

11 Dancsok AR, Setsu N, Gao D, et al. Expression of lymphocyte immunoregulatory biomarkers in bone and soft-tissue sarcomas. Modern Pathology 2019;32:1772-85 https://www-nature-com. proxy.bib.uottawa.ca/articles/s41379-019-0312-y

12 van Erp AEM, Versleijen-Jonkers YMH, Hillebrandt-Roeffen MHS, et al. Expression and clinical association of programmed cell death-1, programmed death-ligand-1 and CD8+ lymphocytes in primary sarcomas is subtype dependent. Oncotarget 2017;8:71371-84.

13 Pollack SM, He Q, Yearley JH, et al. T-Cell infiltration and clonality correlate with programmed cell death protein 1 and programmed death-ligand 1 expression in patients with soft tissue sarcomas. cancer. 2017/05/02. John Wiley and Sons Inc 2017;123:3291-304.

14 Pollack S, Redman MW, Wagner M, et al. A phase I/II study of pembrolizumab (Pem) and doxorubicin (Dox) in treating patients with metastatic/unresectable sarcoma. Journal of Clinical Oncology 2019;37:11009.

15 Momen S, Fassihi H, Davies HR. Dramatic response of metastatic cutaneous angiosarcoma to an immune checkpoint inhibitor in a patient with xeroderma pigmentosum: whole-genome sequencing AIDS treatment decision in end-stage disease. cold spring Harb Mol case stud. Cold Spring Harbor Laboratory Press 2019;5.
16 Conley AP, Trinh VA, Zobniw CM, et al. Positive tumor response to combined checkpoint inhibitors in a patient with refractory alveolar soft part sarcoma: a case report. J Glob Oncol 2018;4:1-6.

17 Marcrom S, De Los Santos JF, Conry RM. Complete response of mediastinal clear cell sarcoma to pembrolizumab with radiotherapy. Clin Sarcoma Res 2017:7.

18 Guram K, Nunez M, Einck J, et al. Radiation therapy combined with checkpoint blockade immunotherapy for metastatic undifferentiated pleomorphic sarcoma of the maxillary sinus with a complete response. Front Oncol 2018;8.

19 George S, Miao D, Demetri GD, et al. Loss of PTEN is associated with resistance to anti-PD-1 checkpoint blockade therapy in metastatic uterine leiomyosarcoma. Immunity 2017;46:197-204.

20 Tawbi HA, Burgess M, Bolejack V, et al. Pembrolizumab in advanced soft-tissue sarcoma and bone sarcoma (SARC028): a multicentre, two-cohort, single-arm, open-label, phase 2 trial. Lancet Oncol 2017;18:1493-501.

21 Keung EZ, Burgess M, Salazar R, et al. Correlative analyses of the SARC028 trial reveal an association between sarcoma-associated immune infiltrate and response to pembrolizumab. Clinical Cancer Research 2020;26:1258-66 https://clincancerres-aacrjournals-org. proxy.bib.uottawa.ca/content/26/6/1258

22 Petitprez F, de Reyniès A, Keung EZ, et al. B cells are associated with survival and immunotherapy response in sarcoma. Nature 2020;577:556-60.

23 Burgess MA, Bolejack V, Schuetze S, et al. Clinical activity of pembrolizumab $(P)$ in undifferentiated pleomorphic sarcoma (ups) and dedifferentiated/pleomorphic liposarcoma (LPS): final results of SARC028 expansion cohorts. Journal of Clinical Oncology 2019;37:11015

24 D'Angelo SP, Mahoney MR, Van Tine BA, et al. Nivolumab with or without ipilimumab treatment for metastatic sarcoma (alliance A091401): two open-label, non-comparative, randomised, phase 2 trials. Lancet Oncol 2018;19:416-26.

25 Chen JL, Mahoney MR, George S, et al. A multicenter phase II study of nivolumab +/- ipilimumab for patients with metastatic sarcoma (alliance A091401): results of expansion cohorts. Journal of Clinical Oncology 2020;38:11511.

26 Keung EZ, Lazar AJ, Torres KE, et al. Phase II study of neoadjuvant checkpoint blockade in patients with surgically resectable undifferentiated pleomorphic sarcoma and dedifferentiated liposarcoma. BMC Cancer 2018;18:913 https://bmccancer. biomedcentral.com/articles/

27 Keung EZ, Tsai J-W, Ali AM, et al. Analysis of the immune infiltrate in undifferentiated pleomorphic sarcoma of the extremity and trunk in response to radiotherapy: rationale for combination neoadjuvant immune checkpoint inhibition and radiotherapy. Oncoimmunology 2018;7:e1385689.

28 Roland CL, Keung EZ-Y, Lazar AJ, et al. Preliminary results of a phase II study of neoadjuvant checkpoint blockade for surgically resectable undifferentiated pleomorphic sarcoma (ups) and dedifferentiated liposarcoma (DDLPS). Journal of Clinical Oncology 2020;38:11505

29 Wilky BA, Trucco MM, Subhawong TK, et al. Axitinib plus pembrolizumab in patients with advanced sarcomas including alveolar soft-part sarcoma: a single-centre, single-arm, phase 2 trial. Lancet Oncol. 2019;20:837-48.

30 Zhao L, Cao YJ. Engineered T cell therapy for cancer in the clinic. Front Immunol 2019;10:2250.

31 Huang R, Li X, He Y, et al. Recent advances in CAR-T cell engineering. J Hematol Oncol 2020;13:86 https://jhoonline. biomedcentral.com/articles/

32 Akers SN, Odunsi K, Karpf AR. Regulation of cancer germline antigen gene expression: implications for cancer immunotherapy. Future Oncol 2010;6:717-32 https://pubmed.ncbi.nlm.nih.gov/ 20465387/

33 Kakimoto T, Matsumine A, Kageyama S, et al. Immunohistochemical expression and clinicopathological assessment of the cancer testis antigens NY-ESO-1 and MAGE-A4 in high-grade soft-tissue sarcoma. Oncol Lett 2019;17:3937-43 https://pubmed.ncbi.nlm.nih. gov/30881511/

34 lura $\mathrm{K}$, Kohashi K, Ishii T, et al. MAGEA4 expression in bone and soft tissue tumors: its utility as a target for immunotherapy and diagnostic marker combined with NY-ESO-1. Virchows Archiv 2017;471:383-92 https://pubmed.ncbi.nlm.nih.gov/28744588/

35 Robbins PF, Kassim SH, Tran TLN, et al. A pilot trial using lymphocytes genetically engineered with an NY-ESO-1-reactive T-cell receptor: long-term follow-up and correlates with response. Clinical Cancer Research 2015;21:1019-27.

36 D'Angelo SP, Melchiori L, Merchant MS, et al. Antitumor activity associated with prolonged persistence of adoptively transferred NY- 
ESO-1 in synovial Sarcoma. Cancer Discov 2018;8:944-57 https:// pubmed.ncbi.nlm.nih.gov/29891538/

37 Ramachandran I, Lowther DE, Dryer-Minnerly R, et al. Systemic and local immunity following adoptive transfer of NY-ESO-1 spear T cells in synovial sarcoma. J Immunother Cancer 2019;7:7 https:// pubmed.ncbi.nlm.nih.gov/31651363/

38 Carvajal RD, Agulnik M, Ryan CW, et al. Trivalent ganglioside vaccine and immunologic adjuvant versus adjuvant alone in metastatic sarcoma patients rendered disease-free by surgery: a randomized phase 2 trial. Journal of Clinical Oncology 2014;32:10520.

39 Dobrenkov K, Ostrovnaya I, Gu J, et al. Oncotargets GD2 and GD3 are highly expressed in sarcomas of children, adolescents, and young adults. Pediatr Blood Cancer 2016;63:1780-5.

40 Long AH, Highfill SL, Cui Y, et al. Reduction of MDSCs with all-trans retinoic acid improves CAR therapy efficacy for sarcomas. Cancer Immunol Res 2016;4:869-80.

41 Majzner RG, Theruvath JL, Nellan A, et al. Car T cells targeting B7$\mathrm{H} 3$, a pan-cancer antigen, demonstrate potent preclinical activity against pediatric solid tumors and brain tumors. Clin Cancer Res 2019;25:2560-74 https://clincancerres.aacrjournals.org/content/ early/2019/01/17/1078-0432.CCR-18-0432

42 Xiao W, Wang J, Wen X, et al. Chimeric antigen receptor-modified T-cell therapy for platelet-derived growth factor receptor $\alpha$-positive rhabdomyosarcoma. Cancer 2020;126:2093-100 https:// onlinelibrary.wiley.com/doi/abs/

43 Tabak SA, Khalifa SE, Fathy Y. Her-2 immunohistochemical expression in bone sarcomas: a new hope for osteosarcoma patients. Open Access Maced J Med Sci 2018;6:1555-60.

44 Chulanetra M, Morchang A, Sayour E, et al. Gd2 chimeric antigen receptor modified $T$ cells in synergy with sub-toxic level of doxorubicin targeting osteosarcomas. Am J Cancer Res 2020;10:674-87 http://www.ncbi.nlm.nih.gov/pubmed/32195035

45 Charan M, Dravid P, Cam M, et al. GD2-directed CAR-T cells in combination with HGF-targeted neutralizing antibody (AMG102) prevent primary tumor growth and metastasis in Ewing sarcoma. Int J Cancer 2020;146:3184-95 https://onlinelibrary.wiley.com/doi/ abs/

46 Merker M, Pfirrmann V, Oelsner S, et al. Generation and characterization of ErbB2-CAR-engineered cytokine-induced killer cells for the treatment of high-risk soft tissue sarcoma in children. Oncotarget 2017;8:66137-53.

47 Leuci V, Casucci GM, Grignani G, et al. Cd44V6 as innovative sarcoma target for CAR-redirected CIK cells. Oncoimmunology 2018;7:7 https://pubmed.ncbi.nlm.nih.gov/29721373/

48 Ahmed N, Brawley V, Hegde M, et al. Her2-Specific chimeric antigen receptor-modified virus-specific $T$ cells for progressive glioblastoma: a phase 1 dose-escalation trial. JAMA Oncol 2017:3:1094-101.

49 Tanaka M, Tashiro H, Omer B, et al. Vaccination Targeting Native Receptors to Enhance the Function and Proliferation of Chimeric Antigen Receptor (CAR)-Modified T Cells. Clinical Cancer Research 2017;23:3499-509.

50 Ray AK, Somanchi SS, Dastgheyb N. Expression of carcinoma, apoptosis, and cell-death-related genes are determinants for sensitivity of pediatric cancer cell lines to lysis by natural killer cells.. Pediatr Blood Cancer 2019:66 https://onlinelibrary.wiley.com/ doi/abs/

51 Vela M, Bueno D, González-Navarro P, et al. Anti-CXCR4 antibody combined with activated and expanded natural killer cells for sarcoma immunotherapy. Front Immunol 2019;10:10.

52 Ojo EO, Sharma AA, Liu R, et al. Membrane bound IL-21 based NK cell feeder cells drive robust expansion and metabolic activation of NK cells. Sci Rep 2019;9:1.

53 Canter RJ, Grossenbacher SK, Foltz JA, et al. Radiotherapy enhances natural killer cell cytotoxicity and localization in preclinical canine sarcomas and first-in-dog clinical trial. J Immunother Cancer 2017;5:98 http://jitc.bmj.com/lookup/doi/

54 Suen WC-W, Lee WY-W, Leung K-T, et al. Natural killer cell-based cancer immunotherapy: a review on 10 years completed clinical trials. Cancer Invest 2018;36:431-57 https://www-tandfonline-com. proxy.bib.uottawa.ca/doi/abs/

55 Kiany S, Gordon N. Aerosol delivery of interleukin-2 in combination with adoptive transfer of natural killer cells for the treatment of lung metastasis: Methodology and effect. Methods Mol Biol [Internet]. Humana Press Inc 2016:285-95 https://pubmed.ncbi.nlm.nih.gov/ 27177675/

56 Kawaguchi S, Tsukahara T, Ida K, et al. Syt-Ssx breakpoint peptide vaccines in patients with synovial sarcoma: a study from the Japanese musculoskeletal Oncology Group. Cancer Sci 2012;103:1625-30 https://pubmed.ncbi.nlm.nih.gov/22726592/
57 Fedorova L, Zdrazilova Dubska L, Pilatova K. 57P Immunomonitoring of patients treated with personalized dendritic cell-based vaccine 58P Influenza vaccine indication during anticancer therapy with immune-checkpoint inhibitors: A transversal challenge for patient's counselling-preliminary analysis of the INVIDla study. Annals of Oncology 2017.

58 Fedorova L, Mudry P, Pilatova K. Assessment of immune response following dendritic cell-based immunotherapy in pediatric patients with relapsing sarcoma. Front Oncol 2019:9 https://pubmed.ncbi. nlm.nih.gov/31799177/

59 Miwa S, Nishida H, Tanzawa Y, et al. Phase 1/2 study of immunotherapy with dendritic cells pulsed with autologous tumor lysate in patients with refractory bone and soft tissue sarcoma. Cancer [Internet].. John Wiley and Sons Inc 2017;123:1576-84 http://doi.wiley.com/

60 Nowicki TS, Berent-Maoz B, Cheung-Lau G, et al. A pilot trial of the combination of transgenic NY-ESO-1-reactive adoptive cellular therapy with dendritic cell vaccination with or without ipilimumab. Clin Cancer Res 2019.

61 Huijbers EJM, van Beijnum JR, Lê CT, et al. An improved conjugate vaccine technology; induction of antibody responses to the tumor vasculature. Vaccine 2018;36:3054-60.

62 Spiegel AM, Gierschik P, Levine MA, et al. Clinical implications of guanine nucleotide-binding proteins as receptor-effector couplers. N Engl J Med 1985;312:26-33 https://pubmed.ncbi.nlm.nih.gov/ 2856889/

63 Paglino JC, van den Pol AN. Vesicular stomatitis virus has extensive oncolytic activity against human sarcomas: rare resistance is overcome by blocking interferon pathways. J Virol 2011;85:9346-58

64 Paglino JC, Andres W, van den Pol AN. Autonomous parvoviruses neither stimulate nor are inhibited by the type I interferon response in human normal or cancer cells. J Virol 2014;88:4932-42 https:// pubmed.ncbi.nlm.nih.gov/24554651/

65 Geiss C, Kis Z, Leuchs B, et al. Preclinical testing of an oncolytic parvovirus: standard protoparvovirus $\mathrm{H}-1 \mathrm{PV}$ efficiently induces osteosarcoma cell lysis in vitro. Viruses 2017:9:301.

66 Lacroix J, Kis Z, Josupeit R, et al. Preclinical testing of an oncolytic parvovirus in Ewing sarcoma: Protoparvovirus $\mathrm{H}-1$ induces apoptosis and lytic infection in vitro but fails to improve survival in vivo. Viruses 2018;10:302-15.

67 Conry RM, Westbrook B, McKee S, et al. Talimogene laherparepvec: first in class oncolytic virotherapy. Hum Vaccin Immunother 2018;14] :839-46 https://pubmed.ncbi.nlm.nih.gov/29420123/

68 Streby KA, Geller JI, Currier MA, et al. Intratumoral injection of HSV1716, an oncolytic herpes virus, is safe and shows evidence of immune response and viral replication in young cancer patients. Clinical Cancer Research 2017;23:3566-74 https://pubmed.ncbi. nIm.nih.gov/28495911/

69 Phan M, Watson MF, Alain T, et al. Oncolytic viruses on drugs: achieving higher therapeutic efficacy. ACS Infect Dis 2018:4:1448-67.

70 Selman M, Ou P, Rousso C, et al. Dimethyl fumarate potentiates oncolytic virotherapy through NF-kB inhibition. Sci Trans/ Med 2018;10:eaao1613 https://pubmed.ncbi.nlm.nih.gov/29367345/

71 Zamarin D, Ricca JM, Sadekova S. Pd-L1 in tumor microenvironment mediates resistance to oncolytic immunotherapy. $J$ Clin Invest 2018.

72 Selman M, Rousso C, Bergeron A, et al. Multi-Modal potentiation of oncolytic virotherapy by vanadium compounds. Mol Ther 2018;26:56-69

73 Navid F, Santana VM, Barfield RC. Anti-Gd2 antibody therapy for GD2-expressing tumors. Curr Cancer Drug Targets 2010;10:200-9 https://pubmed.ncbi.nlm.nih.gov/20201786/

74 Kushner BH, Cheung IY, Modak S, et al. Humanized 3F8 anti-GD2 monoclonal antibody dosing with granulocyte-macrophage colonystimulating factor in patients with resistant neuroblastoma: a phase 1 clinical trial. JAMA Oncol 2018;4:1729-35 https://pubmed.ncbi. nlm.nih.gov/30326045

75 Keyel ME, Reynolds CP. Spotlight on dinutuximab in the treatment of high-risk neuroblastoma: development and place in therapy. Biologics 2019;13:1-12 https://pubmed.ncbi.nlm.nih.gov/30613134/

76 Butch ER, Mead PE, Amador Diaz V, et al. Positron Emission Tomography Detects In Vivo Expression of Disialoganglioside GD2 in Mouse Models of Primary and Metastatic Osteosarcoma. Cancer Res 2019;79:3112-24 https://pubmed.ncbi.nlm.nih.gov/ 31015228/

77 Zhu X, Yang J, Gao Y, et al. The dual effects of a novel peptibody on angiogenesis inhibition and M2 macrophage polarization on sarcoma. Cancer Lett 2018;416:1-10. 
78 Zang $\mathrm{X}$, Zhang $\mathrm{X}, \mathrm{Hu} \mathrm{H}$, et al. Targeted delivery of zoledronate to tumor-associated macrophages for cancer immunotherapy. $\mathrm{Mol}$ Pharm 2019;16:2249-58.

79 Feng $Y$, Mu R, Wang Z, et al. A Toll-like receptor agonist mimicking microbial signal to generate tumor-suppressive macrophages. Nat Commun 2019;10.

80 Zhang W, Huang Q, Xiao W, et al. Advances in anti-tumor treatments targeting the CD47/SIRP $\alpha$ axis. Front Immunol 2020;11:18.

81 Dancsok AR, Gao D, Lee AF, et al. Tumor-Associated macrophages and macrophage-related immune checkpoint expression in sarcomas. Oncoimmunology 2020;9:1747340.

82 Mohanty S, Yerneni K. Theruvath JL, et al. Nanoparticle enhanced MRI can monitor macrophage response to CD47 mAb immunotherapy in osteosarcoma. Cell Death Dis 2019;10:36 http:// www.nature.com/articles/s41419-018-1285-3

83 Ansell S, Chen RW, Flinn IW, et al. A phase 1 study of TTI-621, a novel immune checkpoint inhibitor targeting CD47, in patients with relapsed or refractory hematologic malignancies. Blood 2016;128:1812.

84 Berraondo P, Sanmamed MF, Ochoa MC, et al. Cytokines in clinical cancer immunotherapy. Br J Cancer 2019;120:6-15 https:// pubmed.ncbi.nlm.nih.gov/30413827/

85 Schneider JW, Dittmer DP. Diagnosis and treatment of Kaposi sarcoma. Am J Clin Dermatol 2017;18:529-39.

86 Krown SE. Aids-Associated Kaposi's sarcoma: is there still a role for interferon alfa? Cytokine Growth Factor Rev 2007;18:395-402.

87 Colak S, Ten Dijke P. Targeting TGF- $\beta$ signaling in cancer. Trends Cancer 2017;3:56-71 https://pubmed.ncbi.nlm.nih.gov/28718426/

88 Yeh H-W, Lee S-S, Chang C-Y, et al. A new switch for TGF $\beta$ in cancer. Cancer Res 2019;79:3797-805 https://pubmed.ncbi.nlm. nih.gov/31300476/

89 Lamora A, Talbot J, Bougras G, et al. Overexpression of Smad7 blocks primary tumor growth and lung metastasis development in osteosarcoma. Clinical Cancer Research 2014;20:5097-112 https:// pubmed.ncbi.nlm.nih.gov/25107916/

90 Lamora A, Talbot J, Mullard M, et al. TGF- $\beta$ Signaling in Bone Remodeling and Osteosarcoma Progression. J Clin Med 2016;5:96 https://pubmed.ncbi.nlm.nih.gov/27827889/

91 Chen Y, Zhang K, Li Y, et al. Estrogen-Related receptor $\alpha$ participates transforming growth factor- $\beta$ (TGF- $\beta$ ) induced epithelial-mesenchymal transition of osteosarcoma cells. Cell Adh Migr 2017;11:338-46 https://pubmed.ncbi.nlm.nih.gov/27532429/

92 Saito M, Ichikawa J, Ando T, et al. Platelet-Derived TGF- $\beta$ induces tissue factor expression via the Smad3 pathway in osteosarcoma cells. J Bone Miner Res 2018;33:2048-58 https://pubmed.ncbi.nlm. nih.gov/29949655/

93 Tanyildiz HG, Kaygusuz G, Unal E, et al. The prognostic importance of TGF- $\beta$, TGF- $\beta$ receptor, and fascin in childhood solid tumors. Pediatr Hematol Oncol [Internet]. Taylor and Francis Ltd 2017;34:238-53 https://pubmed.ncbi.nlm.nih.gov/29065267/

94 Neuzillet C, Tijeras-Raballand A, Cohen R, et al. Targeting the TGF $\beta$ pathway for cancer therapy. Pharmacol Ther 2015;147:22-31 https://pubmed.ncbi.nlm.nih.gov/25444759/

95 Matsuzaki J, Gnjatic S, Mhawech-Fauceglia P, et al. Tumorinfiltrating NY-ESO-1-specific CD8 ${ }^{+} \mathrm{T}$ cells are negatively regulated by LAG-3 and PD-1 in human ovarian cancer. Proc Natl Acad Sci U S A 2010;107:7875-80 https://pubmed.ncbi.nlm.nih.gov/20385810/

96 Liu Z, Ravindranathan R, Kalinski P, et al. Rational combination of oncolytic vaccinia virus and PD-L1 blockade works synergistically to enhance therapeutic efficacy. Nat Commun 2017;8:1-12 www. nature.com/naturecommunications

97 Kelly CM, Antonescu CR, Bowler T, et al. Objective response rate among patients with locally advanced or metastatic sarcoma treated with Talimogene Laherparepvec in combination with pembrolizumab: a phase 2 clinical trial. JAMA Oncol 2020;6:402-8 https://pubmed-ncbi-nlm-nih-gov.proxy.bib. uottawa.ca/31971541/

98 D'Angelo SP, Conley AP, Kelly CM, et al. Pilot study of NKTR214 and nivolumab in patients with sarcomas. Journal of Clinical Oncology 2019;37:11010.

99 Somaiah N, Conley AP, Lin HY, et al. A phase II multi-arm study of durvalumab and tremelimumab for advanced or metastatic sarcomas. Journal of Clinical Oncology 2020;38:11509.

100 Ayers M, Lunceford J, Nebozhyn M, et al. IFN- $\gamma$-related mRNA profile predicts clinical response to PD-1 blockade. J Clin Invest 2017:127:2930-40 https://doi.org/

101 Danaher P, Warren S, Lu R, et al. Pan-Cancer adaptive immune resistance as defined by the tumor inflammation signature (TIS): results from the cancer genome atlas (TCGA). J Immunother Cancer 2018;6:63 https://pubmed.ncbi.nlm.nih.gov/29929551 


\section{Correction: Immunotherapy for sarcomas: new frontiers and unveiled opportunities}

Birdi HK, Jirovec A, Cortés-Kaplan S, et al. Immunotherapy for sarcomas: new frontiers and unveiled opportunities. J Immunother Cancer 2021;9:e001580. doi:10.1136/jitc2020-001580.

This article has been corrected since it was first published. The overall response rate for the trial by Kelly et al (reference 97 ) was cited incorrectly and has now been corrected to $35 \%(7 / 20)$.

Open access This is an open access article distributed in accordance with the Creative Commons Attribution Non Commercial (CC BY-NC 4.0) license, which permits others to distribute, remix, adapt, build upon this work non-commercially, and license their derivative works on different terms, provided the original work is properly cited, appropriate credit is given, any changes made indicated, and the use is non-commercial. See http://creativecommons.org/licenses/by-nc/4.0/.

(C) Author(s) (or their employer(s)) 2022. Re-use permitted under CC BY-NC. No commercial re-use. See rights and permissions. Published by BMJ.

J Immunother Cancer 2022;10:e001580corr1. doi:10.1136/jitc-2020-001580corr1

A) Check for updates 